\title{
Asymmetric Behavior of Thymidylate Synthase Dimer Subunits in Denaturating Solvent Observed with Molecular Dynamics
}

\author{
Filip Leonarski, ${ }^{1,2}$ Monika Świniarska, ${ }^{1}$ and Andrzej Les ${ }^{1}$ \\ ${ }^{1}$ Quantum Chemistry Laboratory, Faculty of Chemistry, University of Warsaw, 02-093 Warsaw, Poland \\ ${ }^{2}$ Centre of New Technologies, University of Warsaw, 02-097 Warsaw, Poland \\ Correspondence should be addressed to Filip Leonarski; f.leonarski@cent.uw.edu.pl
}

Received 15 December 2014; Revised 22 February 2015; Accepted 1 March 2015

Academic Editor: Mihaly Mezei

Copyright (c) 2015 Filip Leonarski et al. This is an open access article distributed under the Creative Commons Attribution License, which permits unrestricted use, distribution, and reproduction in any medium, provided the original work is properly cited.

\begin{abstract}
A molecular dynamics simulations of the thymidylate synthase denaturation in chaotrope solvents (urea, guanidinium hydrochloride) were performed on $600 \mathrm{~ns}$ timescale. It appeared that this dimeric enzyme undergoes partial unfolding asymmetrically. It was shown also that urea is a better denaturant in the MD condition, as compared to guanidinium chloride. The unfolding occurs first at the external helices (AA 88-118) and follows by the AA 188-200 region. The present results correspond to the suggested in the literature activity of thymidylate synthase through a half-the-site mechanism.
\end{abstract}

\section{Introduction}

The thymidylate synthase enzyme has been studied experimentally for a long time due to its unique role as an agent catalyzing reductive methylation of dUMP using 5,10methylenetetrahydrofolate as a cofactor [1]. A distinctive feature of thymidylate synthase is that it is the sole de novo source of thymidylate in a cell, which makes it the target protein in various types of chemotherapies [2-4].

Thymidylate synthase (TS) is a dimeric enzyme. There is also an interesting feature of TS. Two structurally equivalent active sites of TS display apparent functional nonequivalence suggesting TS to be a half-the-sites activity enzyme [5]. The principle of half-the-site reactivity was established by Levitzki and coworkers [6] summarizing the discoveries in the late 1960s of many enzymes exhibiting this phenomenon. By this term they meant that the proteins composed of identical subunits with $n$ potential active sites, reacted with a substrate or an inhibitor so that only $n / 2$ sites were occupied when enzyme was saturated with the ligand.

TS undergoes unfolding and denaturation under the action of chaotropes like urea or guanidine hydrochloride (also guanidinium chloride, usually abbreviated as $\mathrm{GdmCl}$ or $\mathrm{GuHCl})$. The early studies have shown that chaotropeinduced unfolding can result in the formation of monomeric species under the action of about $6 \mathrm{M}$ urea and $4 \mathrm{M}$ guanidine hydrochloride solutions [7-9]. Asymmetric behavior, if observed under denaturating conditions, could improve understanding of the half-the-site mechanism.

Chaotrope denaturation mechanism was thoroughly examined for polipeptides and proteins using a computer simulation method, the molecular dynamics (MD) [10-20]. In this method time evolution of an atomistic structure of a protein and surrounding solvent molecules is studied, by solving Newton equation of motions. Potential energy in MD is defined by a so called force field, which contains equations and parameters to find the energy. Applicability range of MD is however limited by currently available hardware resources.

Aim of the already performed chaotrope MD denaturation studies was describing transition states and unfolded states. Achieving it with standard MD protocols was too expensive, so a way to accelerate the method had to be found. Some of the referenced studies use elevated temperatures or increased denaturant concentrations to allow system to quicker crossing of conformational barriers and in result faster unfolding. There are also studies using enhanced sampling [20], where biomolecular system is simulated in parallel in multiple temperatures or states (different level of unfolding). These different states can be exchange under 
certain conditions, allowing much better probing conformational space exploration.

These methods come however with a price of lowering simulation fidelity. Our work is focused on early steps of unfolding and we wanted to describe sequence of events that starts denaturation, rather than getting into unfolded state. Therefore we have decided to use a standard MD protocol, without unphysiological temperatures or enhanced sampling.

In this work we present a study of TS enzyme using MD in water and chaotrope denaturants. We show that denaturation conditions result in asymmetric unfolding of the units and on urea example we give insight into mechanism of first step of TS unfolding.

\section{Methods}

2.1. System Preparation. Thymidylate synthase from rat crystallographic structure was taken from the PDB database (PDB ID: 2TSR [1, 21]). The structure was resolved using X-ray diffraction at $2.6 \AA$ resolution. Crystallographic structure is a dimer of dimers, that is, it consists of two equivalent copies of the enzyme. These copies are later referred to as $\mathrm{AB}$ (first dimer, chains $\mathrm{A}$ and $\mathrm{B}$ ) and $\mathrm{CD}$ (second dimer, chains $\mathrm{C}$ and $\mathrm{D})$. The structure further used in MD simulations was stripped of ligands (Tomudex drug and dUMP), however crystal water molecules were preserved.

Solvent mixture environments for the MD simulations were prepared using a Packmol application [22]. Boxes of $80 \AA \times 80 \AA \times 80 \AA$ in size were filled with solvent molecules according to expected concentrations. Required densities for $8 \mathrm{M}$ urea and $6 \mathrm{M}$ guanidinium chloride solutions in $310 \mathrm{~K}\left(37^{\circ} \mathrm{C}\right)$ were calculated using the empirical equations presented by Kawahara and Tanford [23] as well as tables with water densities depending on temperature.

For a model of $8 \mathrm{M}$ urea solution, the box was composed of 10963 water molecules and 2467 urea molecules; the values proceed from calculated density of $1.1132 \mathrm{~g} / \mathrm{cm}^{3}$ and correspond to 0.750 grams of urea per gram of water, which matches well to the experimental value (0.755) [24]. With calculated $\mathrm{GuHCl}$ solution density equals $1.1363 \mathrm{~g} / \mathrm{cm}^{3}$, a box containing 1850 guanidinium ion molecules $\left(\mathrm{Gu}^{+}\right.$, accompanied by chloride anions) and 9638 water molecules was generated, corresponding to 1.018 grams of denaturant per gram of water, which is in good agreement with the experimental value (1.009) [24]. The boxes were subsequently minimized in NAMD [25] for $500 \mathrm{ps}$, followed by heating (from $0 \mathrm{~K}$ to $310 \mathrm{~K}$ ), and proceeded by $10 \mathrm{~ns}$ molecular dynamics simulation.

The PDB model of dimeric protein of rat thymidylate synthase was immersed in each of the prepared boxes and all solvent molecules within $1.5 \AA$ of the protein were removed, excluding crystal water. As water is much smaller in size than guanidinium ion/urea molecule, the aforementioned procedure of solvent removal was biased towards lowering non-aqueous solvents concentrations. The system was later neutralized with sodium cations, since TS in these $\mathrm{pH}$ conditions is negatively charged; also removal of some $\mathrm{Gu}^{+}$ ions during protein insertion needs to be complemented by cations. Six simulation systems were created: simulation of $\mathrm{AB}$ dimer in water, urea, and guanidinium chloride, as well as $\mathrm{CD}$ dimer in water, urea, and guanidinium chloride. We have performed independent simulation with two different dimer structures to reduce bias from starting coordinates.

2.2. Molecular Dynamics Setup. During the MD simulation the system was described with the use of the CHARMM22 force field $[26,27]$, water molecules were represented by the three-site TIP3P model [28], urea parameters were characterized by Caflisch and Karplus [29], and guanidinium ion parameters were taken from Kuczera [26]; parameters for both nonaqueous solvents were consistent with the CHARMM22 force field. Periodic boundary conditions were used with the Particle Mesh Ewald method [30] to account for long-range electrostatic interactions, with grid spacing of less than $1 \AA$. Simulations were performed using the NAMD 2.8 [25] engine on an IBM BlueGene/P supercomputer.

The minimization of the system internal energy was performed in three steps, each for 100,000 steps: (i) only solvent and ions, (ii) solvent, ions and $\mathrm{C}_{\alpha}$ protein atoms, and (iii) the full system. This was followed by actual MD simulation with $1 \mathrm{fs}$ time step: heating, equilibration, and production. Heating was performed in constant volume conditions until $310 \mathrm{~K}$ temperature was reached, $0.8 \mathrm{ps}$ for every $10 \mathrm{~K}$ step, and giving 24.8 ps run and followed by a 3.2 ps equilibration run at $310 \mathrm{~K}$. These steps were followed by a production run of hundreds of nanoseconds, performed in NPT ensemble at $310 \mathrm{~K}$, with temperature stabilized using a Langevin thermostat (dumping coefficient of $5 \mathrm{ps}^{-1}$ ) and pressure set using a Langevin piston [31, 32]. Langevin method used for keeping temperature and pressure constant are stochastic methods, introducing random fluctuations to the system. Therefore subsequent trajectories of the same system might differ [33]. Accounting for this fluctuation is possible with having all simulations in all the solvent conditions performed with either $\mathrm{AB}$ or $\mathrm{CD}$ dimer.

2.3. Denaturant Concentrations. Setting pressure constant in the simulation accounts for imperfections of structure preparation. If any cavities were left after inserting the protein to a solvent box, the periodic boundary conditions box will be compacted to account for that. However, due to variable volume, the simulation is in principle not a constant concentration simulation (number of solvent molecules do not change with volume fluctuations).

Taking this into account, as well as effects of insertion of the protein to the box effective concentrations in this study, come to ca. $5.9 \mathrm{M}$ for urea and $3.8 \mathrm{M}$ for $\mathrm{GuHCl}$, at the beginning of simulation. Being consistent with experimental values of $6 \mathrm{M}$ urea and $4 \mathrm{M} \mathrm{GuHCl}$ provided in the Introduction.

2.4. Trajectory Analysis. The trajectories obtained during the simulation were analyzed using the Cpptraj application, 

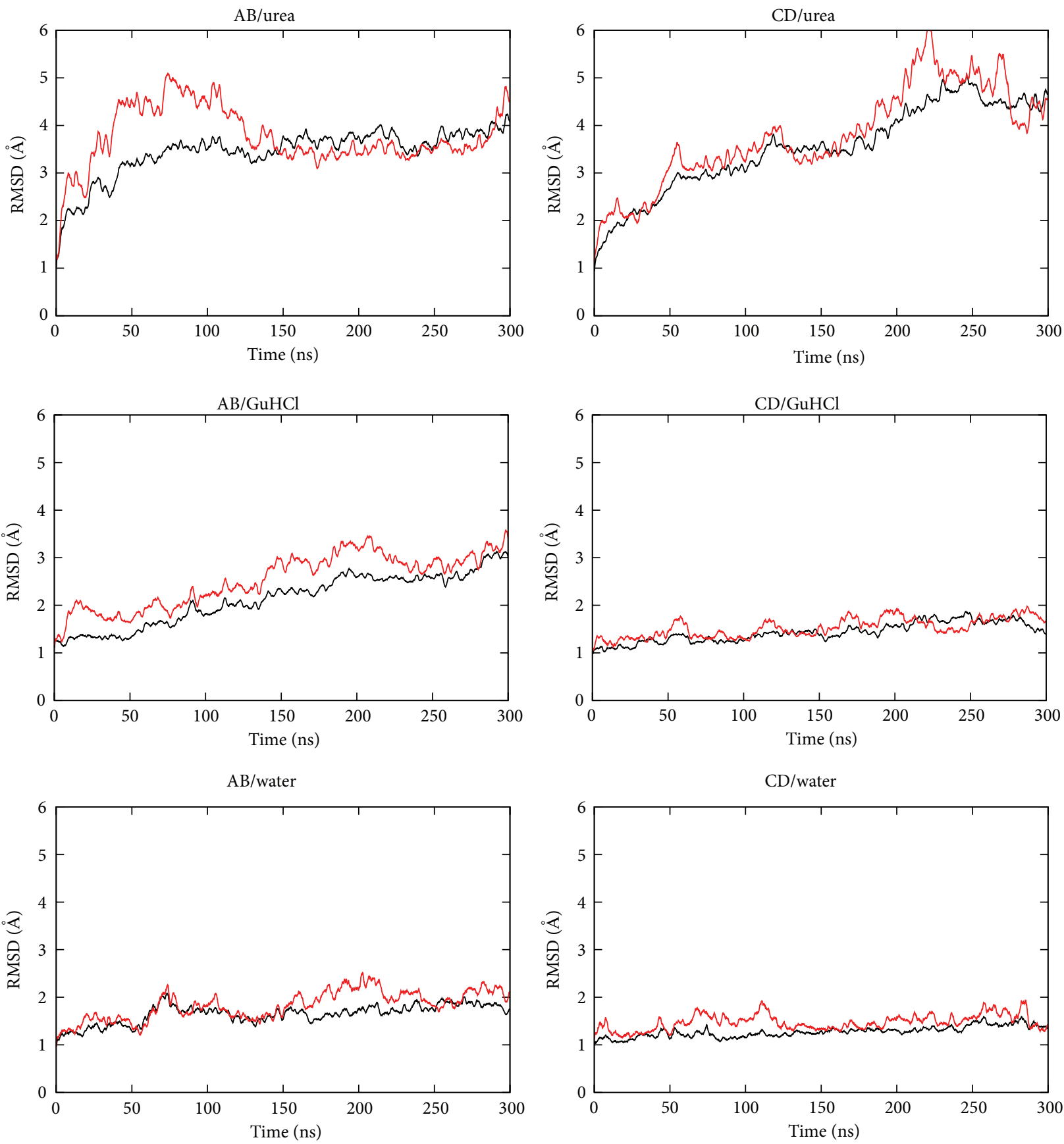

FIGURE 1: Root mean square deviation in reference to starting structure (black) calculated between $\mathrm{C}_{\alpha}$ carbon coordinates of the first dimeric subunit versus the second subunit (intermonomer RMSD red) of the thymidylate synthase in function of time for 300 ns molecular dynamics simulation. Raise of the intermonomer RMSD value shows asymmetric unfolding of the subunits, especially in case of urea.

available in the AmberTools 13 suite $[34,35]$. The following aspects of the simulation were taken into consideration.

(i) Root Mean Square Deviation (RMSD): A measure of similarity of molecule 3D-fold to a reference structure, usually crystallographic one, is routinely used in analyzing MD simulations to find stability of a molecule in time. If $\mathbf{x}_{i}(t)$ is the $i$ th (out of $N$ ) atom position after time $t$, and $\mathbf{x}_{i}^{\text {ref }}$ is value of the atom position in a reference molecule, then RMSD is defined as

$$
\operatorname{RMSD}(t)=\sqrt{\frac{1}{N} \sum_{i=1}^{N}\left(\mathbf{x}_{i}(t)-\mathbf{x}_{i}^{\mathrm{ref}}\right)^{2}} .
$$

RMSD value is minimized over all possible translations and rotations of the superimposed molecule. In 

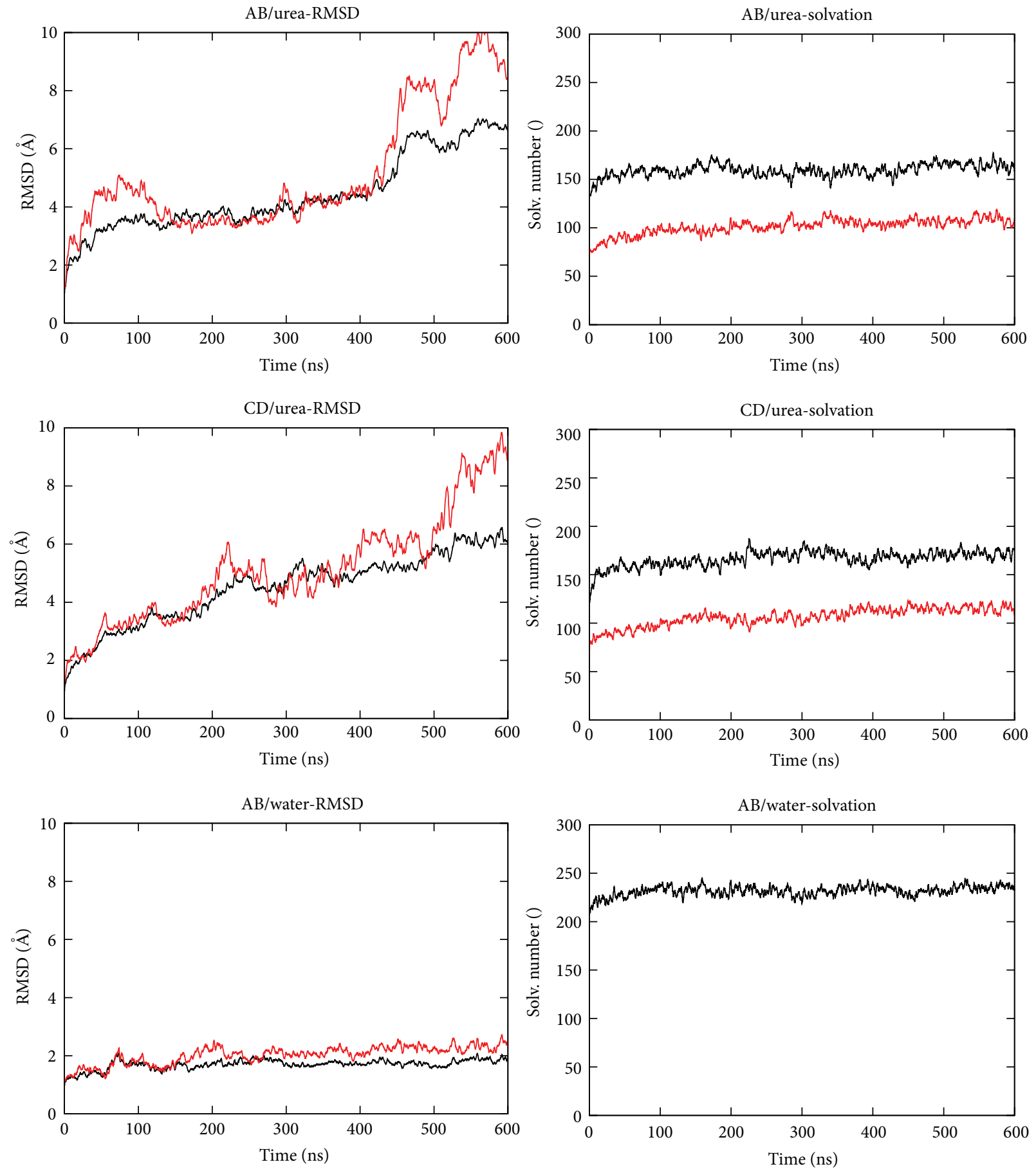

FIGURE 2: Comparison of RMSD (left side, black), intermonomer RMSD (left side, red), solvation number of water (right side, black), and urea (right side, red). Values were processed with the moving average method (window of 20 points) to decrease noise.

this paper we consider RMSD calculated only for $\mathrm{C}_{\alpha}$ atom positions.

(ii) Intermonomer $R M S D$ : A measure of asymmetry between dimer units of the protein in function of time. In each time step RMSD value is calculated between the first and the second monomer unit coordinates (coordinates of the second monomer at time $t$ substitute reference position in (1)). Coordinates are translated and rotated using Kabsch algorithm [36] before RMSD calculation to minimize RMSD value.

(iii) Root Mean Square Fluctuation (RMSF): A measure of mobility of atoms/residues during a period of time. If $\mathbf{x}_{i}(t)$ is the $i$ th atom position (out of $N$ ) after $t$ 
time steps (out of $T$ ), and $\mathbf{x}_{i}^{\mathrm{ref}}$ is the $i$ th atom position reference value, then RMSF is defined as

$$
\operatorname{RMSF}(i)=\sqrt{\frac{1}{T} \sum_{t=1}^{T}\left(\mathbf{x}_{i}(t)-\mathbf{x}_{i}^{\mathrm{ref}}\right)^{2}} .
$$

As in case of RMSD, in this paper, we report RMSF of only $\mathrm{C}_{\alpha}$ atoms to remove contributions from internal movements in amino acids. The reference value of atom position is its average position over time. Structures were superimposed to the reference (starting) structure according to the lowest RMSD before calculating RMSF.

(iv) Solvation Number: The number of solvent molecules occupying space in the $5 \AA$ cut-off distance from the solute.

\section{Results and Discussion}

3.1. Effect of Solvent Choice on the Thymidylate Synthase Structure. Initially we have simulated all 6 solute/solvent pairs on 300 ns time scale. General observation from RMSD measurements (Figure 1) is that the protein in water remains stable, so it can be taken as a reference for chaotrope simulations. There are ongoing changes in conformation in the urea solution. Guanidinium hydrochloride solutions shows also signs of denaturation, but much slower. Progress of denaturation is slow, when compared with smaller molecule studies.

In terms of asymmetric behavior in Figure 1 we present intersubunit RMSD data for TS simulations. In water simulations the intersubunit RMSD remains at level of $2 \AA$. For guandinium hydrochloride in one simulation the value raised to more than $3 \AA$, but in other simulation remained at $2 \AA$ level. Finally in case of urea the value raised to 5-6 $\AA$ level.

\subsection{In-Depth Analysis of Thymidylate Synthase AB Dimer} Denaturation in Urea Solution. As $300 \mathrm{~ns}$ simulation of thymidylate synthase in urea revealed signs of ongoing denaturation, we have chosen to continue a longer simulation and we have been able to run MD for twice as long (600 ns) for the following systems: (a) AB dimer in urea, (b) CD dimer in urea, and (c) $\mathrm{AB}$ dimer in water. The trajectories allowed to perform an in-depth analysis of the obtained results to answer questions about initial steps of denaturation.

Comparison of the $600 \mathrm{~ns}$ trajectories is presented in Figure 2. Global trend for the three trajectories is not different from that of the $300 \mathrm{~ns}$ simulations. The protein remains stable in water while denaturation in urea continues, as seen on the RMSD plot.

Also intermonomer RMSD continues to rise to level of approximately $10 \AA$ by the end of a simulation time. This value is higher than RMSD of the whole protein calculated from starting structure, in range of $6 \AA$ by the end of simulation. On the other hand there is no visible sign of increasing solvation of the protein, as seen on model systems simulations [20].

To approach the problem profoundly, local measures have to be taken into account. Figure 3 presents RMSF changes
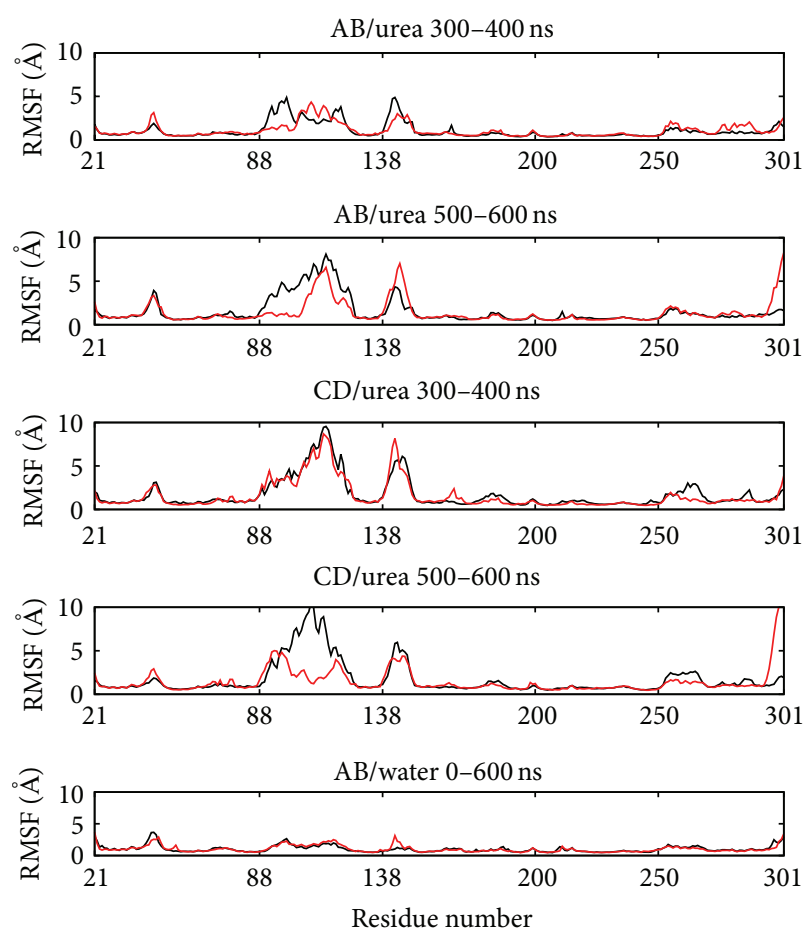

FIGURE 3: RMSF of the protein in urea solution during different parts of trajectory. Black line corresponds to RMSF of the first monomer (A or C) and red line corresponds to RMSF of the second monomer (B or D). Since the simulation in water did not show signs of structure evolution, the whole trajectory of the protein in water is taken as a reference. Residues are numbered according to the protein sequence, with certain residues not present in the PDB structure (i.e., amino acids 1-20 and 302-307).

during the simulation of the protein in urea, as compared to simulation in water. Figure 4, on the other hand, presents how secondary structure of the protein is affected by denaturing conditions. The regions with the highest observed flexibility are $\alpha$-helices between amino acids 88 and 138. In both A and $\mathrm{B}$ monomers one can observe decrease in percentage of time in which amino acids are in helical conformation (see Figure 4). General picture is similar, however careful observation of atoms mobility reveals that between 400 and 500 ns subunit A shows approximately 1.5 higher values of fluctuations than the B one (see Figure 3: level of 12-13 $\AA$ for the monomer $\mathrm{A}$ and maximum value of $8 \AA$ for the monomer B). On the other hand, the region between amino acids 138 and 150 is occupied in the monomer $\mathrm{A}$ by anti-parallel $\beta$ strands, which are not present in the monomer B. Careful analysis of Figure 4 might even suggest that these anti-parallel strands in the B subunit are slowly developing in the progress of the simulation. In case of the A subunit there is also a small increase in fluctuations in $3_{10}$-helix between residues 180 and 200. This region is an inner part of the protein, close to the active site. It is possible that large fluctuations in the A88-138 region result in noticeable movements of A180-200 region, and since fluctuations in the B88-138 are smaller in magnitude, they do not affect B180-200 area. The positions 


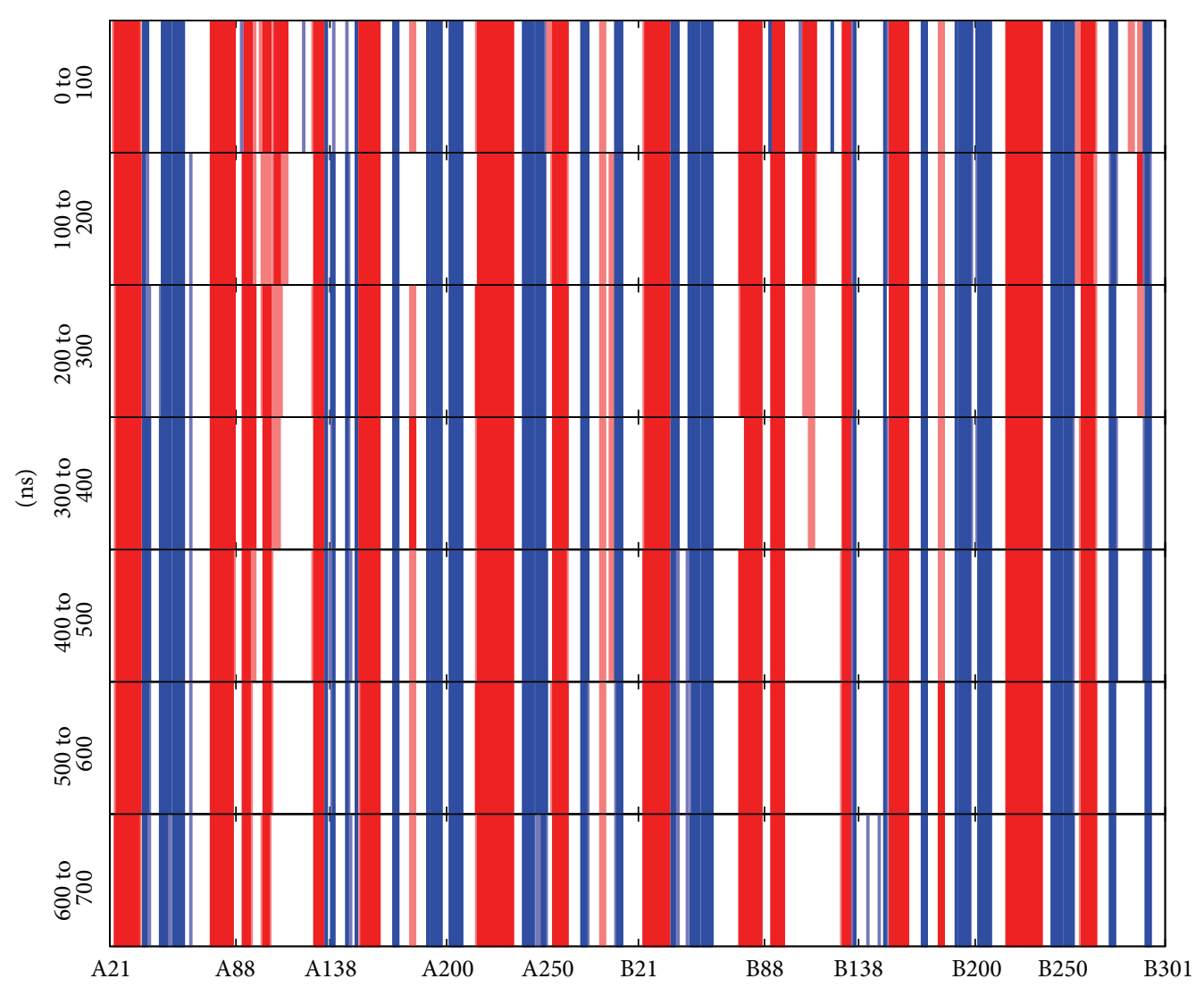

FIGURE 4: The evolution of the secondary structure of the AB dimer of thymidylate synthase in urea solution calculated using DSSP [38] algorithm for different intervals of the trajectory. Presented are helical areas (red) and $\beta$ strands (blue). Darker color represents secondary structure element present in at least $75 \%$ of frames in the interval; lighter color represents element present in $25-75 \%$ of frames. Residues are numbered according to the protein sequence, with certain residues not present in the PDB structure (i.e., amino acids 1-20 and 302-307).

of the most flexible regions in the course of initial steps of denaturation are visualized in Figure 5.

\section{Conclusions}

In this work we have shown that denaturation of a relatively large protein, like thymidylate synthase, in adverse solvent conditions, might be observed in molecular dynamics simulations on hundreds of nanosecond timescales $(0.5 \mu \mathrm{s})$. Shorter simulations, requiring less computational effort would not be able to catch even a limited scope of the denaturation. The fact that the protein remained stable in similar simulation setup, but only with pure water, allows to show that urea and guanidine hydrochloride used in simulations are responsible for denaturation effects seen in Figures 1-4.

However early signs of denaturation where seen in the simulation, simulation time shorter than a microsecond is not enough to capture full denaturation pathway of the protein. Similar timescales were successful to observe denaturation for small polipeptides, like a lysozyme [19], which has more than 5 times less residues than the thymidylate synthase dimer. Even case of such smaller proteins only partial denaturation pathway was observed during 1 microseond. Therefore, as expected our trajectory does not give answers on unfolding pathway of the internal core, including the active site.

When analyzing denaturation of the protein monomers only denaturation of external loops can be seen in the trajectories. According to the results, unfolding of the external helices comprising the amino acids from 88 to 118 is the first step in the denaturation process of thymidylate synthase, later accompanied by unfolding of the 188-200 region. Since the latter region is close to the active site, we suspect that this is where denaturation of the active site is beginning. Preferential location of 88-118 amino acids as well as 138-150 ones on the exterior of the protein (see Figure 5) makes them ideal areas for denaturation to start at. There is no surprise they show very high atoms mobility (see Figure 3), followed by losing their secondary structures in the progress of the simulation (see Figure 4).

The denaturation process ran asymmetrically with the greater changes and faster unfolding appearing in one subunit rather than in its counterpart. This is similar to the proposed mechanism of the dimer activity, where only one monomer is active at the time [37]. The results we obtained do not prove the half-of-the-sites activity of TS, rather they show unequivalent unfolding of monomeric subunits which may 


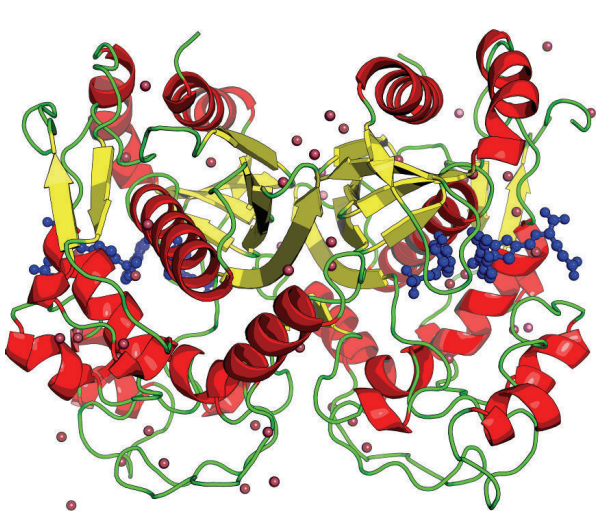

(a)

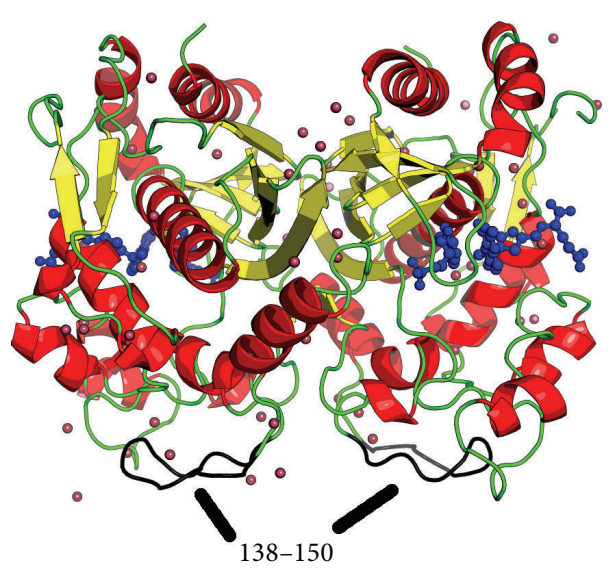

(c)

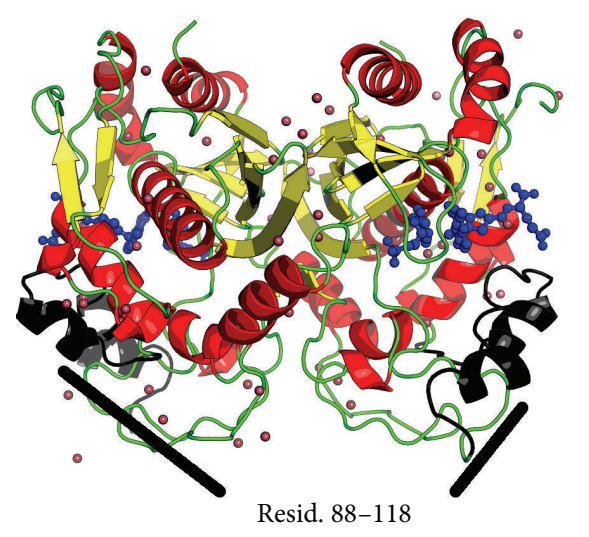

(b)

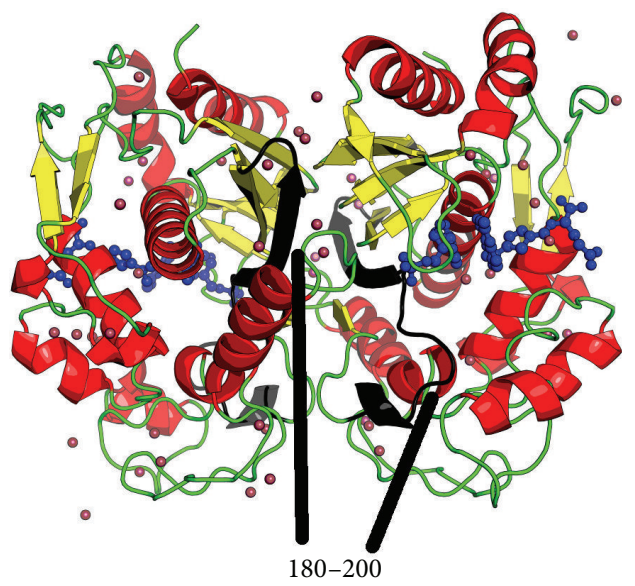

(d)

Figure 5: (a) Thymidylate synthase dimer 3D structure, colors represent 2D folds: red- $\alpha$-helix, yellow- $\beta$-sheet, green-loops, blue molecule-ligands in the active site (not present in MD), and red beads-water. (b) TS structure with residues 88-118 (in both dimeric units) highlighted in black. (c) TS structure with residues 138-150 (in both dimeric units) highlighted in black. (d) TS structure with residues 180-200 (in both dimeric units) highlighted in black.

form a molecular basis for the half-reduced activity of the dimer.

\section{Conflict of Interests}

The authors declare that there is no conflict of interests regarding the publication of this paper.

\section{Acknowledgments}

The authors acknowledge computational time provided by ICM supercomputer center (G18-6) on Notos IBM BlueGene/P supercomputer maintained under the POWIEW project "HPC Infrastrcture for Grand Challenges of Science and Engineering," cofinanced by the European Union from the European Regional Development Fund. The authors thank Dr. Nina Pastor (Universidad Autonoma del Estado de Morelos, Mexico), Dr. Bartosz Trzeskowski, and Piotr Broda for help in the early stage of the work. Filip Leonarski also thanks Dr. Joanna Trylska, Joanna Panecka, and Katarzyna
Kulczycka-Mierzejewska for useful help in setting up and analyzing MD simulations.

\section{References}

[1] R. R. Sotelo-Mundo, J. Ciesla, J. M. Dzik et al., "Crystal structures of rat thymidylate synthase inhibited by tomudex, a potent anticancer drug," Biochemistry, vol. 38, no. 3, pp. 1087-1094, 1999.

[2] P. V. Danenberg, “Thymidylate synthetase: a target enzyme in cancer chemotherapy," Biochimica et Biophysica Acta, vol. 473, no. 2, pp. 73-92, 1977.

[3] L. W. Hardy, J. S. Finer-Moore, W. R. Montfort, M. O. Jones, D. V. Santi, and R. M. Stroud, "Atomic structure of thymidylate synthase: target for rational drug design," Science, vol. 235, no. 4787, pp. 448-455, 1987.

[4] B. K. Shoichet, R. M. Stroud, D. V. Santi, I. D. Kuntz, and K. M. Perry, "Structure-based discovery of inhibitors of thymidylate synthase," Science, vol. 259, no. 5100, pp. 1445-1450, 1993.

[5] F. Maley, J. Pedersen-Lane, and L. Changchien, "Complete restoration of activity to inactive mutants of Escherichia coli 
thymidylate synthase: evidence that $E$. coli thymidylate synthase is a half-the-sites activity enzyme," Biochemistry, vol. 34, no. 5, pp. 1469-1474, 1995.

[6] A. Levitzki, W. B. Stallcup, and D. E. Koshland Jr., "Half-ofthe-sites reactivity and the conformational states of cytidine triphosphate synthetase," Biochemistry, vol. 10, no. 18, pp. 33713378, 1971.

[7] R. S. Gokhale, S. Agarwalla, D. V. Santi, and P. Balaram, "Covalent reinforcement of a fragile region in the dimeric enzyme thymidylate synthase stabilizes the protein against chaotrope-induced unfolding," Biochemistry, vol. 35, no. 22, pp. 7150-7158, 1996.

[8] L. A. May, H. C. Cheung, J. L. Aull, and J. D. Glickson, "Fluorescence study of guanidine hydrochloride denaturation of thymidylate synthetase," Biochemical and Biophysical Research Communications, vol. 73, no. 3, pp. 653-658, 1976.

[9] J. W. Reinsch, L. L. Smith, and R. B. Dunlap, "Denaturation of thymidylate synthetase from amethopterin-resistant Lactobacillus casei," Cancer Biochemistry Biophysics, vol. 3, no. 2, pp. 57-64, 1979.

[10] J. Tirado-Rives, M. Orozco, and W. L. Jorgensen, "Molecular dynamics simulations of the unfolding of barnase in water and $8 \mathrm{M}$ aqueous urea," Biochemistry, vol. 36, no. 24, pp. 7313-7329, 1997.

[11] A. Caflisch and M. Karplus, "Structural details of urea binding to barnase: a molecular dynamics analysis," Structure, vol. 7, no. 5, pp. 477-488, 1999.

[12] Z. Zhang, Y. Zhu, and Y. Shi, "Molecular dynamics simulations of urea and thermal-induced denaturation of S-peptide analogue," Biophysical Chemistry, vol. 89, no. 2-3, pp. 145-162, 2001.

[13] D. Tobi, R. Elber, and D. Thirumalai, "The dominant interaction between peptide and urea is electrostatic in nature: a molecular dynamics simulation study," Biopolymers, vol. 68, no. 3, pp. 359369, 2003.

[14] B. J. Bennion and V. Daggett, "The molecular basis for the chemical denaturation of proteins by urea," Proceedings of the National Academy of Sciences of the United States of America, vol. 100, no. 9, pp. 5142-5147, 2003.

[15] B. J. Bennion and V. Daggett, "Counteraction of urea-induced protein denaturation by trimethylamine N-oxide: a chemical chaperone at atomic resolution," Proceedings of the National Academy of Sciences of the United States of America, vol. 101, no. 17, pp. 6433-6438, 2004.

[16] D. K. Klimov, J. E. Straub, and D. Thirumalai, "Aqueous urea solution destabilizers $\mathrm{A} \beta_{16-22}$ oligomers," Proceedings of the National Academy of Sciences of the United States of America, vol. 101, no. 41, pp. 14760-14765, 2004.

[17] A. Caballero-Herrera, K. Nordstrand, K. D. Berndt, and L. Nilsson, "Effect of urea on peptide conformation in water: molecular dynamics and experimental characterization," Biophysical Journal, vol. 89, no. 2, pp. 842-857, 2005.

[18] V. Daggett, "Protein folding-simulation," Chemical Reviews, vol. 106, no. 5, pp. 1898-1916, 2006.

[19] L. Hua, R. Zhou, D. Thirumalai C, and B. J. Berne, "Urea denaturation by stronger dispersion interactions with proteins than water implies a 2-stage unfolding," Proceedings of the National Academy of Sciences of the United States of America, vol. 105, no. 44, pp. 16928-16933, 2008.

[20] R. Meloni, C. Camilloni, and G. Tiana, "Sampling the denatured state of polypeptides in water, urea, and guanidine chloride to strict equilibrium conditions with the help of massively parallel computers," Journal of Chemical Theory and Computation, vol. 10, no. 2, pp. 846-854, 2014.

[21] H. M. Berman, T. Battistuz, T. N. Bhat et al., "The protein data bank," Acta Crystallographica Section D: Biological Crystallography, vol. 58, no. 6, pp. 899-907, 2002.

[22] L. Martínez, R. Andrade, E. G. Birgin, and J. M. Martínez, "Packmol: a package for building initial configurations for molecular dynamics simulations," Journal of Computational Chemistry, vol. 30, no. 13, pp. 2157-2164, 2009.

[23] K. Kawahara and C. Tanford, "Viscosity and density of aqueous solutions of urea and guanidine hydrochloride," The Journal of Biological Chemistry, vol. 241, no. 13, pp. 3228-3232, 1966.

[24] C. N. Pace and J. M. Scholtz, "Measuring the conformational stability of a protein," Protein Structure: A practical Approach, vol. 2, pp. 299-321, 1997.

[25] J. C. Phillips, R. Braun, W. Wang et al., "Scalable molecular dynamics with NAMD," Journal of Computational Chemistry, vol. 26, no. 16, pp. 1781-1802, 2005.

[26] A. D. MacKerell Jr., D. Bashford, M. Bellott et al., "All-atom empirical potential for molecular modeling and dynamics studies of proteins," The Journal of Physical Chemistry B, vol. 102, no. 18, pp. 3586-3616, 1998.

[27] A. D. Mackerell Jr., M. Feig, and C. L. Brooks III, "Extending the treatment of backbone energetics in protein force fields: limitations of gas-phase quantum mechanics in reproducing protein conformational distributions in molecular dynamics simulation," Journal of Computational Chemistry, vol. 25, no. 11, pp. 1400-1415, 2004.

[28] W. L. Jorgensen, "Transferable intermolecular potential functions for water, alcohols, and ethers. Application to liquid water," Journal of the American Chemical Society, vol. 103, no. 2, pp. 335340, 1981.

[29] A. Caflisch and M. Karplus, "Acid and thermal denaturation of barnase investigated by molecular dynamics simulations," Journal of Molecular Biology, vol. 252, no. 5, pp. 672-708, 1995.

[30] T. Darden, L. Perera, L. Li, and P. Lee, "New tricks for modelers from the crystallography toolkit: the particle mesh Ewald algorithm and its use in nucleic acid simulations," Structure, vol. 7, no. 3, pp. R55-R60, 1999.

[31] G. J. Martyna, D. J. Tobias, and M. L. Klein, "Constant pressure molecular dynamics algorithms," The Journal of Chemical Physics, vol. 101, no. 5, pp. 4177-4189, 1994.

[32] S. E. Feller, Y. Zhang, R. W. Pastor, and B. R. Brooks, "Constant pressure molecular dynamics simulation: the Langevin piston method," The Journal of Chemical Physics, vol. 103, no. 11, pp. 4613-4621, 1995.

[33] P. Auffinger, S. Louise-May, and E. Westhof, "Multiple molecular dynamics simulations of the anticodon loop of trnaasp in aqueous solution with counterions," Journal of the American Chemical Society, vol. 117, no. 25, pp. 6720-6726, 1995.

[34] D. R. Roe and T. E. Cheatham, "PTRAJ and CPPTRAJ: Software for processing and analysis of molecular dynamics trajectory data," Journal of Chemical Theory and Computation, vol. 9, no. 7, pp. 3084-3095, 2013.

[35] D. Case, T. Darden, I. Cheatham et al., AMBER 12, University of California, San Francisco, Calif, USA, 2013.

[36] W. Kabsch, "A solution for the best rotation to relate two sets of vectors," Acta Crystallographica Section A: Crystal Physics, Diffraction, Theoretical and General Crystallography, vol. 32, part 5, pp. 922-923, 1976. 
[37] W. R. Montfort, K. M. Perry, E. B. Fauman et al., "Structure, multiple site binding, and segmental accommodation in thymidylate synthase on binding dUMP and an anti-folate," Biochemistry, vol. 29, no. 30, pp. 6964-6977, 1990.

[38] W. Kabsch and C. Sander, "Dictionary of protein secondary structure: pattern recognition of hydrogen-bonded and geometrical features," Biopolymers, vol. 22, no. 12, pp. 2577-2637, 1983. 

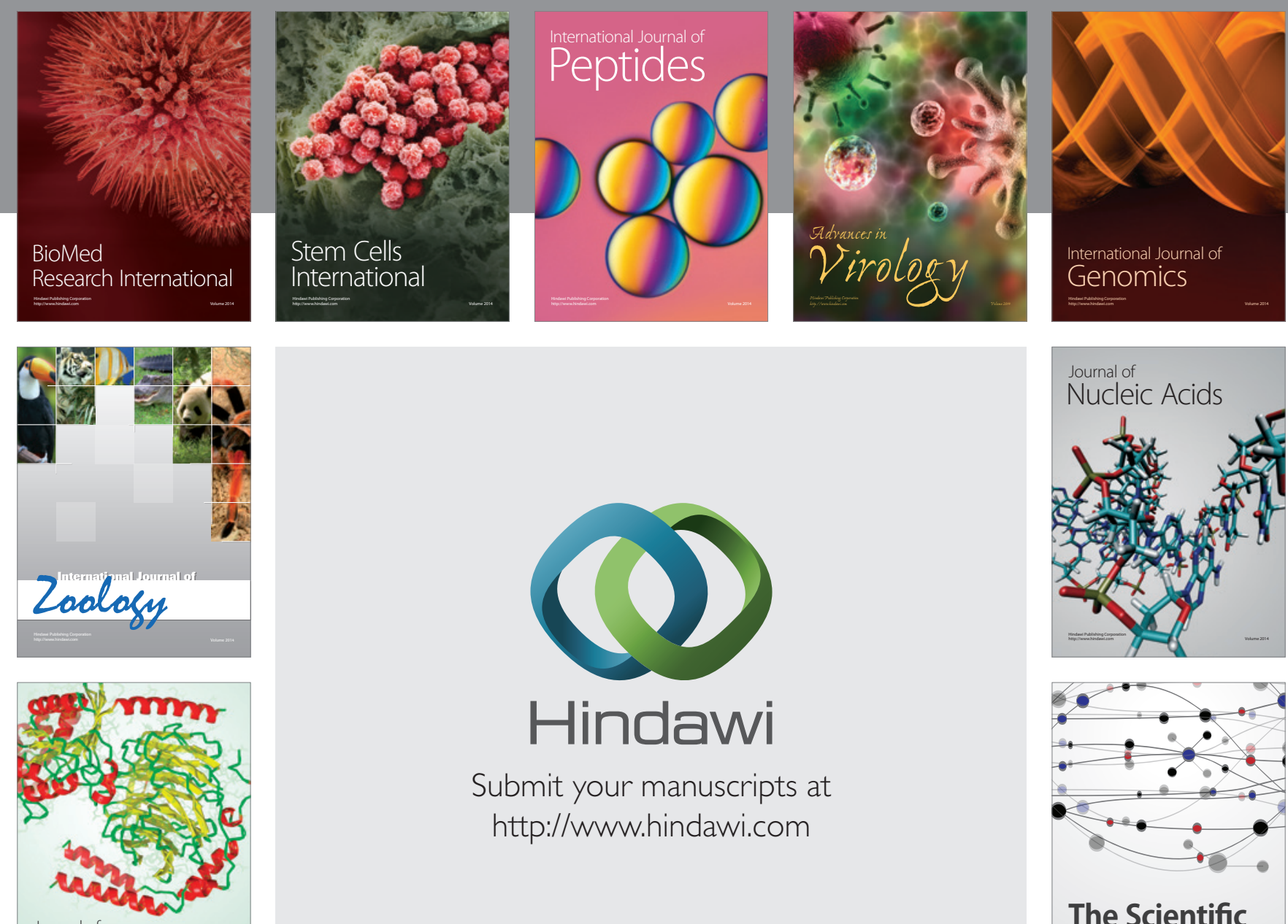

Submit your manuscripts at

http://www.hindawi.com

Journal of
Signal Transduction
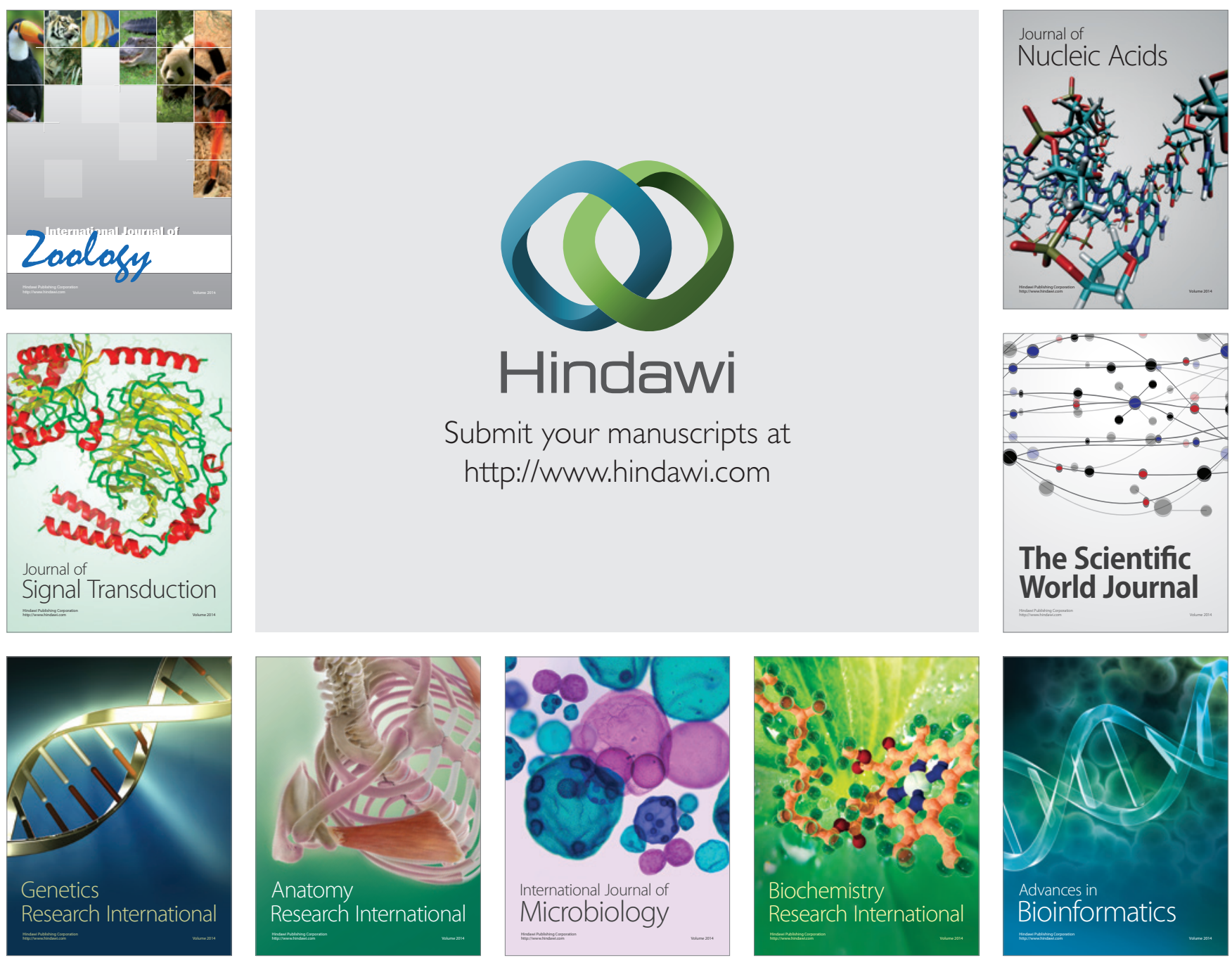

The Scientific World Journal
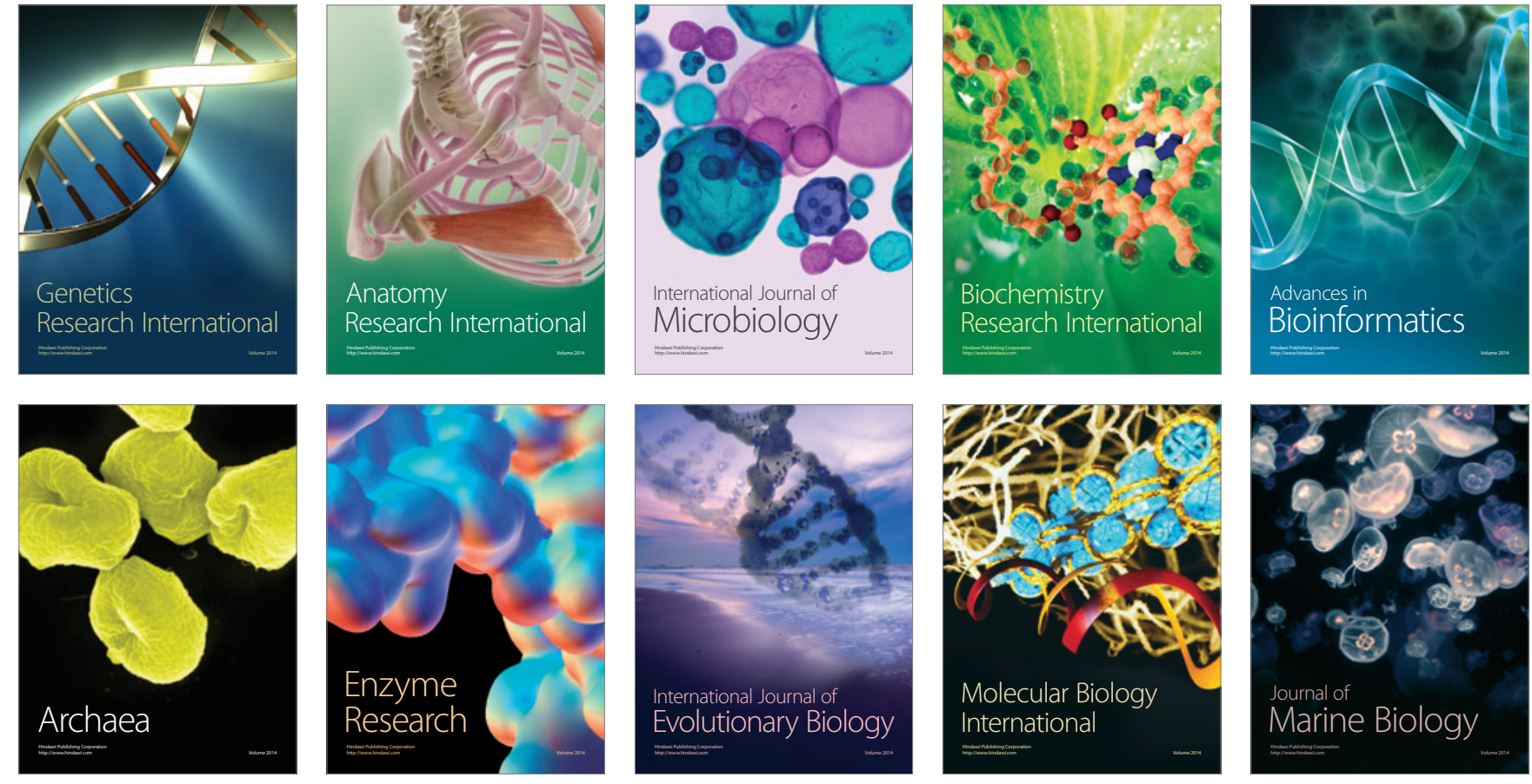